\title{
THE FLUXION IN A CURVED JOSEPHSON JUNCTION
}

\section{TOMASZ DOBROWOLSKI}

\section{Communicated by Jean-Francois Ganghoffer}

\begin{abstract}
The curved Josephson junction is described. In the framework of the Maxwell equations the equation that describes the influence of the curvature on the fluxion motion was obtained. The method of geometrical reduction of the sine-Gordon model from three to lower dimensional manifold was applied to the long Josephson junction. It was argued that the geometrical reduction describes the junctions with slowly varying curvatures.
\end{abstract}

\section{Introduction}

Two superconducting electrodes separated by a thin layer of a dielectric material form the Josephson Junction. The quantum state of each electrode of this system is described by a macroscopic wave function. The modules of these functions measure the square root of the density of Cooper pairs in the superconductors. In a bulk of each electrode, the density of Cooper pairs is almost constant and therefore the bulk the dominating dynamical degrees of freedom are phases of these wave functions. If the central dielectric layer, which separates the electrodes, is sufficiently narrow (typically several of $\AA$ ), then the phases are correlated and the number of independent degrees of freedom is reduced to only one scalar function $\phi$. This function describes a gauge invariant phase difference between superconducting electrodes. The physical effect of this correlation is a flow of Cooper pairs through the dielectric barrier. This effect has been predicted by Josephson [16] and then confirmed experimentally by Anderson and Rowell [2].

Depending on the dimensions of the Josephson device it can be considered as a two, one and even zero dimensional system. In particular, if the transverse dimension of the junction is smaller than the Josephson length, then the system can be considered as the one dimensional system called the long Josephson junction. The behavior of the dynamical variable $\phi$ that describes this system is determined by the sineGordon model [6]. This model describes the dynamics of many other systems [5]. Solutions of this model are extensively studied for years [1], [12]. The most known solution of this model is a kink which interpolates between different ground states 
of the potential. In the context of the long Josephson junction the kink represents a quanta of the magnetic flux called the fluxion.

Technological progress in recent years has enabled the fabrication of arbitrarily curved junctions. On the other hand, there are also theoretical attempts to study the influence of nontrivial geometry on the fluxion motion in such systems. For example, in [14] the authors concentrated on influence of the curved boundaries on the kink motion. The junction, in their example, is located on the plane and it has the form of two rectangles connected by the ring. The curved region of this junction corresponds to the existence of a nontrivial potential that affects the kink motion. Moreover, the influence of the external magnetic field on the annular junction was studied theoretically and experimentally in [15], [23]. In the distant perspective, these studies are motivated by the possibility of construction of quantum computers. The Josephson junctions can be used in these devices as their basic elements, i.e., qubits $[11,13,18,19,24]$. The other possible deformations of the Josephson junction may also correspond to bending of the dielectric layer in an arbitrary way. From geometric point of view, such a curved junction can be represented by a curve or a curved surface nontrivially embedded in a three dimensional space, i.e., its external curvatures are not trivial. On the other hand, the sine-Gordon model that describes the dynamics of the gauge invariant phase difference can be relatively easy projected onto an arbitrary curved manifold. In this situation, the simplest method of construction of the effective model that describes the kink motion in a curved junction is connected with geometric reduction of the sine-Gordon model to lower dimensional curved subspace [9].

In the present work we recognize the limitations of the geometrical reduction method in the context of the Josephson junction.

The paper is organized as follows. In the next Section, we introduce the curved coordinates on the basis of the junction and fix our notation. We also recollect some elements of the method on the example of a plane curve. Section 3 is devoted to construction of the curved field equations on the basis of physical considerations. In the same section we recognize also the regime of applicability of the geometrical reduction method. The last Section contains remarks concerning possible applications of the curved junctions.

\section{Geometrical Reduction of the Sine-Gordon Model}

We first recollect basic facts concerning geometrical reduction of the sine-Gordon model to lower dimensional manifold. In this article, the model is reduced to plane curve located in the center of the insulator layer. The physical context of this 


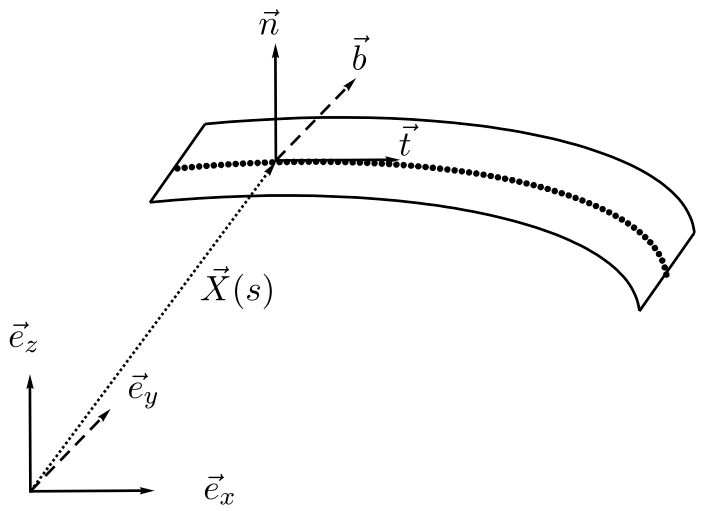

Figure 1. The connection between the Frenet frame $\{\vec{t}, \vec{b}, \vec{n}\}$ on the central curve of the insulator layer and Cartesian coordinates. The $s$ coordinate parameterizes the central curve.

choice is the propagation of the electromagnetic waves in a long and quasi-one dimensional Josephson junction. To consider the model defined by the Lagrangian density

$$
\mathcal{L}=\frac{1}{2}\left(\partial_{t} \phi\right)^{2}-\frac{1}{2} \eta_{E}^{i j}\left(\partial_{i} \phi\right)\left(\partial_{j} \phi\right)-V(\phi)
$$

where $V(\phi)=1-\cos \phi$ is the sine-Gordon potential and $\eta_{E}^{i j}$ is the Euclidean metric in the Cartesian coordinates $\left(x^{i}\right)=\left(x^{1}, x^{2}, x^{3}\right)=(x, y, z)$. For the sake of simplicity, coordinates used in this Section are dimensionless, i.e., $x^{i} \rightarrow x^{i} / \lambda_{J}$ and $t \rightarrow \omega_{P} t$, where $\lambda_{J}$ is the Josephson length and $\omega_{P}$ is the plasma frequency. We also use suitable curved coordinates. The first coordinate $s$ parameterizes the central line of the junction, the second $\rho$ parameterizes the normal (to orientation of $s$ variable) direction of the junction and the third $u$ is normal to the junction. In Fig. 1, the first variable corresponds to the direction of the tangent vector $\vec{t}$, the direction of the second coordinate $\rho$ is indicated by the binormal vector $\vec{b}$, and the last variable is connected with direction of the normal vector $\vec{n}$.

The Lagrangian density in these coordinates is the following

$$
\mathcal{L}=\frac{1}{2}\left(\partial_{t} \phi\right)^{2}-\frac{1}{2} G^{\alpha \beta}\left(\partial_{\alpha} \phi\right)\left(\partial_{\beta} \phi\right)-V(\phi)
$$

where we use the notation $\left(\xi^{\alpha}\right)=\left(\xi^{1}, \xi^{2}, \xi^{3}\right)=\left(s, \rho^{1}, \rho^{2}\right)=(s, \rho, u)$ and also $\left(\rho^{I}\right)=\left(\rho^{1}, \rho^{2}\right)=(\rho, u)$. We identify the junction with a curve located in the center of the insulator layer and therefore we identify its points by the vector field $\vec{X}(s)$. In the present article we presume that the junction is flat in the direction of the $\rho$ variable. In the neighborhood of the central line, there exists a connection of 
curved coordinates with coordinates of the Cartesian reference frame

$$
\vec{x}=\vec{X}(s)+\rho^{I} \vec{n}_{I}(s)
$$

where $\vec{n}_{2} \equiv \vec{n}$ is the normal vector to the central curve and $\vec{n}_{1} \equiv \vec{b}$ is the binormal vector. We also denote the tangent vector to the central line as follows $\partial_{s} \vec{X} \equiv$ $\vec{X}_{, s} \equiv \vec{t}$. Moreover, the vectors $\vec{n}_{I}$ are normalized to unity and are orthogonal each other and to the tangent vector $\vec{X}_{, s}=\vec{t}$ to the central line as well

$$
\vec{n}_{I} \cdot \vec{n}_{J}=\delta_{I J}, \quad \vec{n}_{J} \cdot \vec{X}_{, s}=0
$$

where the scalar products are calculated with respect to the Euclidean metric in Cartesian coordinates. The similar curved coordinates are also used in other physical contexts $[4,8,20]$. In general, an embedding of the line in 3D space is described by the extrinsic curvature $K$ and the torsion coefficient $\omega$. On the other hand, here we assume that the curve is plane and therefore $\omega=0$. In the calculus we shall need the metric in the curved coordinates $\left(\xi^{\alpha}\right)=\left(s, \rho^{I}\right)$

$$
G^{\alpha \beta}=\frac{\partial \xi^{\alpha}}{\partial x^{i}} \frac{\partial \xi^{\beta}}{\partial x^{j}} \eta_{E}^{i j}
$$

The components of this metric are the following

$$
G^{I J}=\delta^{I J}, \quad G^{I s}=0, \quad G^{s s}=\frac{1}{G} .
$$

The determinant of the metric $G_{\alpha \beta}$ in curved coordinates has the form

$$
G=\mathcal{G}^{2}=(1-u K(s))^{2} .
$$

On the intermediate step of the reduction procedure we obtain the Lagrangian density

$$
\mathcal{L}=\frac{1}{2}\left(\partial_{t} \phi\right)^{2}-\frac{1}{2 G}\left(\partial_{s} \phi\right)^{2}-V(\phi)
$$

where we removed derivatives with respect to normal variables $\partial_{\rho} \phi=0$ and $\partial_{u} \phi=0$. The effective Lagrangian density which describes the motion of the kink along the junction can be obtained by integration the Lagrangian density (7) with respect to normal variables (see [9])

$$
L=\int \mathrm{d} s \int_{-w / 2}^{+w / 2} \mathrm{~d} \rho \int_{-a / 2}^{+a / 2} \mathrm{~d} u \sqrt{G} \mathcal{L}=\int \mathrm{d} s \mathcal{L}_{\text {eff }}
$$

where $a$ is thickness and $w$ is width of the isolator layer. After integration we obtain

$$
\mathcal{L}_{e f f}=\frac{1}{2}\left(\partial_{t} \phi\right)^{2}-\frac{1}{2} \mathcal{F}\left(\partial_{s} \phi\right)^{2}-V(\phi)
$$


where we omitted the multiplicative constant $a w$ and introduced a function of curvature as follows

$$
\mathcal{F}=\frac{1}{a K(s)} \ln \left(\frac{2+a K(s)}{2-a K(s)}\right) .
$$

The equation of motion connected with the effective Lagrangian density has the form

$$
\partial_{t}^{2} \phi-\partial_{s}\left(\mathcal{F} \partial_{s} \phi\right)+\sin \phi=0
$$

\section{Physical Explanation of the Influence of Curvature on the Fluxion Dynamics}

In this Section we shall obtain the equations that describe the propagation and generation of electromagnetic waves in the long Josephson junction [22], [6]. The junction, depicted in Fig. 2, consists of two superconducting electrodes separated by the dielectric layer of thickness $a$. In this layer we choose the surface $\Sigma$, presented in Fig. 1, that divides the insulator on two parts and is equally separated from the superconducting electrodes by the distance $a / 2$. On this surface, we chose plane curve parameterized by the $s$ coordinate. This curve is located in the center of the surface $\Sigma$, i.e., is equally separated from the boundaries of the junction by the distance $w / 2$ ( $w$ is a width of the junction). This curve represents geometry of the long Josephson junction. We presume that the surface $\Sigma$ is flat in the direction of the $\rho$ coordinate and also that physical settings exclude any dynamics in this direction. These assumptions allow to apply the obtained results to some class of the two dimensional Josephson junctions, i.e., the quasi-one dimensional large area Josephson junctions. In Fig. 2, one can see, that both superconducting electrodes are penetrated by the electromagnetic field up to the distance of London penetration depths $\left(\lambda_{T}\right.$ and $\left.\lambda_{B}\right)$. The doted areas represent parts of the electrodes penetrated by the magnetic field.

In the presence of the vector potential $\vec{A}$ the Landau-Ginzburg electric current density can be written in the form

$$
\vec{J}=\frac{e^{*}}{m^{*}}\left[\frac{1}{2} \mathrm{i} \hbar\left(\psi \nabla \psi^{*}-\psi^{*} \nabla \psi\right)-\frac{e^{*}}{c} \vec{A} \psi \psi^{*}\right]
$$

where $\psi$ is the macroscopic wave function. The effective charge and mass parameters, in the framework of the BCS theory can be interpreted as a charge and the mass of the Cooper pairs and therefore are equated to double the electron charge $e^{*}=2 e$ and mass $m^{*}=2 m$. The density of Cooper pairs in the macroscopic state (described by the modulus of the wave function) is almost constant in the bulk of 


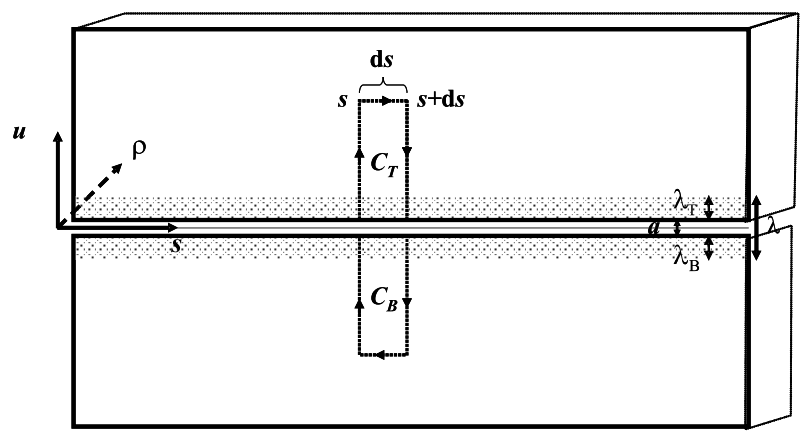

Figure 2. The Josephson junction consists of two superconductors. Each of the superconducting electrodes is penetrated by the magnetic field. The penetration area is represented by doted part of the figure (the penetration depths in top and bottom electrodes are $\lambda_{T}$ and $\lambda_{B}$ ). The thickness of the insulator layer is denoted by $a$. The $s$ coordinate parameterizes the points of the central line of the junction. The $u$ variable indicates the normal direction to the junction.

the superconducting material. The formula (12) can be simplified to the form

$$
\vec{J}=|\psi|^{2} \frac{e}{m}\left[\hbar \nabla \varphi-\frac{2 e}{c} \vec{A}\right]
$$

where $\varphi$ is a phase of the macroscopic wave function $\psi=|\psi| \mathrm{e}^{\mathrm{i} \varphi}$. From this equation one can calculate the gradient of the phase factor

$$
\nabla \varphi=\frac{2 e}{\hbar c}\left[\frac{m c}{2|\psi|^{2} e^{2}} \vec{J}+\vec{A}\right] .
$$

We consider the curved junction and therefore we use the local curved coordinates $(s, \rho, u)$ defined in the vicinity of the central surface of the insulator layer. An arbitrary vector in these coordinates decomposes as follows

$$
\vec{A}=A_{s} \vec{t}+A_{\rho} \vec{b}+A_{u} \vec{n} .
$$

In order to guarantee one dimensionality, we assume that the magnetic field has nonzero component only in the direction of the $\rho$ variable. Moreover, we presume that the fields are homogenous in the direction of the $\rho$ coordinate (i.e., they do not depend on $\rho$ ). In these circumstances we can choose gauge so as the only nonzero component of the gauge potential is $A_{s}$. In these conditions waves propagate in the direction of the $s$ coordinate. In the curved coordinates $(s, \rho, u)$, the gradient of a scalar field takes the form

$$
\nabla V=\frac{1}{\mathcal{G}} \partial_{s} V \vec{t}+\partial_{\rho} V \vec{b}+\partial_{u} V \vec{n}
$$


and the curl of a vector field is the following

$$
\operatorname{rot} \vec{A}=\left[\partial_{\rho} A_{u}-\partial_{u} A_{\rho}\right] \vec{t}+\frac{1}{\mathcal{G}}\left[\partial_{u}\left(\mathcal{G} A_{s}\right)-\partial_{s} A_{u}\right] \vec{b}+\frac{1}{\mathcal{G}}\left[\partial_{s} A_{\rho}-\partial_{\rho}\left(\mathcal{G} A_{s}\right)\right] \vec{n}
$$

Our purpose is the calculation of the gauge invariant phase difference between bottom and top electrodes of the junction in infinitesimally separated points $s$ and $s+\mathrm{d} s$. We recollect the $s$ component of the gradient (16)

$$
(\operatorname{grad} \varphi)_{s}=\frac{1}{\mathcal{G}} \partial_{s} \varphi
$$

and then use relation (14) in order to calculate the phase differences in top

$$
\frac{\varphi_{T}(s)}{\mathcal{G}(s)}-\frac{\varphi_{T}(s+\mathrm{d} s)}{\mathcal{G}(s+\mathrm{d} s)}=\frac{2 e}{\hbar c} \int_{C_{T}} \mathrm{~d} \vec{l}\left[\frac{m c}{2|\psi|^{2} e^{2}} \vec{J}+\vec{A}\right]=\frac{2 e}{\hbar c} \int_{C_{T}} \mathrm{~d} \vec{l} \vec{A}
$$

and bottom electrodes

$$
\frac{\varphi_{B}(s+\mathrm{d} s)}{\mathcal{G}(s+\mathrm{d} s)}-\frac{\varphi_{B}(s)}{\mathcal{G}(s)}=\frac{2 e}{\hbar c} \int_{C_{B}} \mathrm{~d} \vec{l}\left[\frac{m c}{2|\psi|^{2} e^{2}} \vec{J}+\vec{A}\right]=\frac{2 e}{\hbar c} \int_{C_{B}} \mathrm{~d} \vec{l} \vec{A} .
$$

The contours of integration $C_{T}$ and $C_{B}$ (see Fig. 2) are chosen deep inside the superconducting electrodes where the shielding current density is equal to zero. Moreover the parts of the contours $C_{T}$ and $C_{B}$ in the area penetrated by the magnetic field (of the size of London penetration depths) are perpendicular to the current $\vec{J}$.

Let us also notice that in the considered situation the electromagnetic field has zero $A_{u}$ component and therefore the gauge invariant difference of the phases can be expressed simply as a difference of the phases in the bottom and top electrodes

$$
\phi(s, t) \equiv \varphi_{B}(s, t)-\varphi_{T}(s, t)+\frac{2 e}{\hbar c} \int_{-a / 2}^{a / 2} \mathrm{~d} u A_{u}=\varphi_{B}(s, t)-\varphi_{T}(s, t) .
$$

If we add formulas (19), (20) and use the definition (21), then we obtain

$$
\frac{\phi(s+\mathrm{d} s)}{\mathcal{G}(s+\mathrm{d} s)}-\frac{\phi(s)}{\mathcal{G}(s)}=\frac{2 e}{\hbar c} \oint \mathrm{d} \vec{l} \vec{A}
$$

where we additionally neglected the thickness of the barrier. From the Stokes theorem, the contour integral can be expressed as a surface integral over the surface spanned by the union of both contours

$$
\frac{\phi(s+\mathrm{d} s)}{\mathcal{G}(s+\mathrm{d} s)}-\frac{\phi(s)}{\mathcal{G}(s)}=\frac{2 e}{\hbar c} \int_{S} \int \mathrm{d} \vec{S} \vec{H}=\frac{2 e}{\hbar c} \lambda \mathrm{d} s H_{\rho}
$$


where $\lambda$ describes the thickness of the region penetrated by the magnetic field $\left(\lambda=a+\lambda_{T}+\lambda_{B}\right.$, here $a$ is a width of the isolator layer and $\lambda_{T}, \lambda_{B}$ are London penetration depths of the top and bottom superconductors). In the zero $\mathrm{d} s$ limit we obtain

$$
\partial_{s}\left(\frac{1}{\mathcal{G}} \phi\right)=\frac{2 e}{\hbar c} \lambda H_{\rho} .
$$

The last equation connects the magnetic field with the gauge invariant phase difference.

On the other hand, we use Maxwell equations written in terms of free charges and currents. In Gaussian units, Ampere's circuital law with Maxwell's correction has the form

$$
\operatorname{rot} \vec{H}=\frac{4 \pi}{c} \vec{J}+\frac{1}{c} \partial_{t} \vec{D} .
$$

The third component of this equation depends on first $H_{s}$ and second $H_{\rho}$ components of the magnetic field

$$
\frac{1}{\mathcal{G}}\left[\partial_{s} H_{\rho}-\partial_{\rho}\left(\mathcal{G} H_{s}\right)\right]=\frac{4 \pi}{c} J_{u}+\frac{1}{c} \partial_{t} D_{u} .
$$

In the described experimental settings the first component of the magnetic field is absent. Moreover, we fixed gauge in such a way that the third component of the vector potential is equal to zero and therefore the electric induction only depends only on the electric potential

$$
D_{u}=\varepsilon E_{u}=\varepsilon\left(-\partial_{u} V-\frac{1}{c} \partial_{t} A_{u}\right)=-\varepsilon \partial_{u} V
$$

where $\varepsilon$ is relative dielectric constant. Next, we integrate the last formula with respect to the normal variable

$$
\int_{-a / 2}^{a / 2} \mathrm{~d} u D_{u}=-\varepsilon \int_{-a / 2}^{a / 2} \mathrm{~d} u \partial_{u} V=\varepsilon(-V(a / 2)+V(-a / 2))=\varepsilon\left(V_{B}-V_{T}\right) .
$$

As a result, we connect the normal component of the electric induction with the potential difference between superconducting electrodes $D_{u}=\frac{1}{a} \varepsilon \Delta V$, where $\Delta V \equiv\left(V_{B}-V_{T}\right)$. Thus we can write the equation (26) as follows

$$
\frac{1}{\mathcal{G}} \partial_{s} H_{\rho}=\frac{4 \pi}{c} J_{u}+\frac{\varepsilon}{a c} \partial_{t}(\Delta V) .
$$

If we combine the Maxwell equation (29) with the second Josephson relation

$$
\partial_{t} \phi=\frac{2 e}{\hbar} \Delta V
$$


and formula (24), we obtain the equation

$$
\frac{\hbar c}{2 e \lambda} \partial_{s}^{2}\left(\frac{1}{\mathcal{G}} \phi\right)=\mathcal{G} \frac{4 \pi}{c} J_{u}+\mathcal{G} \frac{\varepsilon}{a c} \frac{\hbar}{2 e} \partial_{t}^{2} \phi .
$$

Next we average this formula with respect to the normal variable of the junction $\left(\frac{1}{a} \int_{-a / 2}^{+a / 2} \mathrm{~d} u f\right)$

$$
\frac{\hbar c}{2 e \lambda} \partial_{s}^{2}(\mathcal{F} \phi)=\frac{4 \pi}{c} J_{u}+\frac{\varepsilon}{a c} \frac{\hbar}{2 e} \partial_{t}^{2} \phi
$$

In the next step, we use the first Josephson relation $J_{u}=J_{J} \sin \phi$ and obtain the following equation

$$
\frac{\hbar c^{2}}{8 \pi e \lambda} \partial_{s}^{2}(\mathcal{F} \phi)=J_{J} \sin \phi+C \frac{\hbar}{2 e} \partial_{t}^{2} \phi .
$$

Here $C=\frac{\varepsilon}{4 \pi a}$ is the junction capacitance per unit area and $a$ is the width of the dielectric layer. Finally, the equation of motion for gauge invariant phase difference has the form

$$
\frac{1}{\bar{c}^{2}} \partial_{t}^{2} \phi-\partial_{s}^{2}(\mathcal{F} \phi)+\frac{1}{\lambda_{J}^{2}} \sin \phi=0
$$

where the Josephson length is defined as $\lambda_{J}=\sqrt{\frac{\hbar c^{2}}{8 \pi e \lambda J_{J}}}$ and the Swihart velocity is $\bar{c}=\frac{c}{\sqrt{4 \pi \lambda C}}$. In dimensionless units $\left(s \rightarrow \frac{1}{\lambda_{J}} s\right.$ and $\left.t \rightarrow \frac{\bar{c}}{\lambda_{J}} t=\omega_{P} t\right)$, this equation can be simplified in the form

$$
\partial_{t}^{2} \phi-\partial_{s}^{2}(\mathcal{F} \phi)+\sin \phi=0
$$

Let us notice that equation (35) coincides with the equation (11) of the previous Section in the case of constant curvature. For non-constant curvature, this correspondence is approximate provided that the curvature changes sufficiently slowly i.e., $R^{\prime}(s)<<R(s)$ and $R^{\prime \prime}(s)<<R(s)$, where $R(s)$ is radius of curvature and the prime denotes derivative with respect to $s$ variable. For example, the first condition means that the change of the curvature radius $\Delta R$ on the distance $\Delta s$ is much smaller than the product $R \Delta s$.

The more general form of the equation of motion can be obtained if we take into account the more general form of the first Josephson relation [17], [3]

$$
J_{u}=J_{J} \sin \phi+\sigma_{0} V+\sigma_{1} V \cos \phi
$$

where $\sigma_{0}$ and $\sigma_{1}$ are appropriate conductivities of the junction. The first term represents tunneling of the Cooper pairs. The second term of this relation describes the quasiparticle tunneling current and the third a quasiparticle pairs interface current. 
Actually, the last term describes a concomitant destruction and creation of pairs on different sides of the junction. This relation imposed in equation (32) leads to the field equation

$$
\frac{1}{\bar{c}^{2}} \partial_{t}^{2} \phi+\frac{\gamma}{\bar{c}} \partial_{t} \phi+\frac{\beta}{\bar{c}} \cos \phi \partial_{t} \phi-\partial_{s}^{2}(\mathcal{F} \phi)+\frac{1}{\lambda_{J}^{2}} \sin \phi=0
$$

where $\beta=\frac{\sigma_{1}}{\bar{c} C}$ and $\gamma=\frac{\sigma_{0}}{\bar{c} C}$. As parameters $\sigma_{0}$ and $\sigma_{1}$ are usually really small then the new terms are neglected or in other cases can be treated on the ground of a perturbational scheme.

Finally, let us recall that gauge invariant phase difference between superconducting electrodes $\phi$ has a direct physical meaning, i.e., it is interpreted as a normalized measure of the magnetic flux. This interpretation follows from the second Josephson relation that leads to proportionality of the gauge invariant phase difference $\phi$ to the magnetic flux $\Phi$.

\section{Remarks}

In the present article, we have discussed the geometrical reduction method that was used in order to describe the motion of the kink in the arbitrary deformed Josephson junction. The outcome of this method is the collective coordinate effective Lagrangian that describes the dynamics of the adiabatic variable indicating position of the kink.

In the present work, we concentrated on the long Josephson junction represented by a plane curve. The junction of this type, due to small width, is treated as one dimensional system. Moreover, if the magnetic field is chosen in an appropriate way, then the same considerations apply to the large area Josephson junction (that is two dimensional system). In the second case, one have to use the junction with one flat direction.

First, we performed geometrical reduction of the sine-Gordon model to the subspace that has the form of a plane curve. Next, we calculated the effects of curvature on the physical background. Let us notice that even this method has one drawback, i.e., it neglects the magnetic flux located in the dielectric layer. In order to shorten calculus, we compared both results on the level of the field equation which is intermediate step on the way to the collective coordinate effective Lagrangian.

As a result we have obtained precise agreement of two results for junctions with constant curvature. Moreover, the same result was obtained on a more fundamental level [7]. On the other hand, if the curvature is a slowly varying function of the variable $s$ then both results agree approximately. On the physical background, 
we have obtained also equations that take into account two other physical effects. The first is the existence of the quasiparticle tunneling current in the system which introduces the damping term to the equation of motion. The second is a quasiparticle pairs interface current which modifies the sine-Gordon equation by the cosine term. This term is usually very small and therefore can be treated in the framework of suitable constructed perturbational scheme. The approach presented here is based on the macroscopic wave function that is outcome of the BCS theory in the mean-field approximation. The equation obtained in Section 4 has direct application to the description of the transmission lines. In the case of the Josephson junctions with slowly varying curvatures, the description of the system is given by the Lagrangian (9).

The description of curved junctions with slowly varying curvatures have two positive features, which are connected with the existence of a Lagrangian and moreover Hamiltonian formulation of the effective model in this regime. The first feature is existence of the kink solutions, that follows directly from Bogomolny analysis. The second is the possibility to go beyond the mean field approximation. This last feature is based on the collective coordinate approach and could be particularly useful in construction of the qubit. The natural candidate for a collective coordinate is the position of the fluxion identified with the position of zero of the kink ansatz [21]

$$
\phi_{K}=4 \arctan \left[\mathrm{e}^{s-S(t)}\right]
$$

The effective Lagrangian for this variable

$$
L=T-U
$$

contains the kinetic

$$
T=2 a b J(S) \dot{S}^{2} \approx 4 a b \dot{S}^{2}
$$

and the potential energy that, for example, for small curvatures is closely related with curvature itself

$$
U(S) \approx 8 a b+\frac{b a^{3}}{6} \int_{0}^{l} \mathrm{~d} s \frac{K(s)^{2}}{\cosh ^{2}(s-S)} \equiv 8 a b+\Delta U
$$

In this sense the curvature corresponds to the potential barrier and the hole in the system. For example, if the Josephson junction consists of two straight segments $K(s)=0$ for $s \in\left[0, s_{1}\right] \cup\left[s_{2}, l\right]$ connected by the arc of the circle of radius $R$ (i.e., $K(s)=1 / R=$ const for $s \in\left[s_{1}, s_{2}\right]$ ), then the potential energy has a form of the barrier (see Fig. 3). Similarly, if two arc of the circle are separated by the straight segment, then we have a potential hole. 


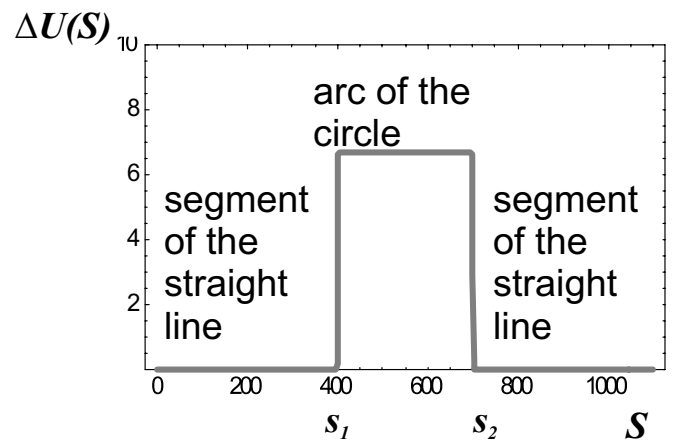

Figure 3. The potential barrier formed in the region of constant curvature (i.e., $K(s)=1 / R$ for $s \in\left[s_{1}, s_{2}\right]$ ). This picture is valid whenever $0<$ $s_{1}<s_{2}<l$. The parameters in the plot are the following $a=20, R=200$, $b=100, l=1000, s_{2}=700, s_{1}=400$.

The equations (35), (37) describe the dynamics of the gauge invariant phase difference in the mean field approximation of the BCS model. The description of a quantum excitations of the system that goes beyond this approximation needs quantization of the collective variable $S$. The quantization of the system can be performed by imposing the canonical commutation relations between the position and conjugate momentum: $[\hat{S}, \hat{P}]=\mathrm{i} \hbar$. The description of the system is now given by a quantum Hamiltonian

$$
\hat{H}=\frac{1}{16 a b} \hat{P}^{2}+U(\hat{S})
$$

where in position representation $\hat{S} \rightarrow S$ and $\hat{P} \rightarrow-\mathrm{i} \hbar \frac{\partial}{\partial S}$. The two lowest energy eigenstates $\psi_{\lambda}(S)$

$$
\hat{H} \psi_{\lambda}(S)=E_{\lambda} \psi_{\lambda}(S)
$$

of the Hamiltonian (42) can be used in order to construct the quantum bit. Let us notice that during the production process of the junction and due to the relation (41) the form of the potential can be chosen in such a way that the two lowest energy states can be well separated from the rest of the eigenvalues of the Hamiltonian (42). Moreover, the equation (41) can help to choose the most suitable form of the potential $U(\hat{S})$ by the proper choice of the shape of the Josephson junction. For example, if for a given form of the potential the solution of the eigenvalue problem (43) is known, then (instead of searching for solutions of the eigenvalue problem) we can concentrate on finding appropriate curvature of the junction. 


\section{Acknowledgements}

This work was supported in part by NCN Grant 2011/03/B/ST3/00448.

\section{References}

[1] Ablowitz M. and Clarkson P., Solitons, Nonlinear Evolution equations and Inverse Scattering, Cambridge Univ. Press, Cambridge 1999.

[2] Anderson P. and Rowell J., Probable Observation of the Josephson Superconducting Tunnelling Effect, Phys. Rev. Lett. 10 (1963) 230-232.

[3] Arnold G., Theory of Tunneling in Superconductors, Phys. Rev. B 17 (1978) 3576-3588.

[4] Arodź H. and Pełka R., Evolution of Interfaces and Expansion in Width, Phys. Rev. E 62 (2000) 6749-6759.

[5] Babelon O., Bernard D. and Talon M., Introduction to Classical Integrable Systems, Cambridge Univ. Press, Cambridge 2003.

[6] Barone A. and Paterno G., Physics and Applications of the Josephson Effect, Wiley, New York 1982.

[7] Dobrowolski T., Curved Josephson Junction, Ann. Phys. NY. 327 (2012) 1336-1354.

[8] Dobrowolski T., Geometry of Vortices and Domain Walls, J. Geom. Symmetry Phys. 22 (2011) 1-12.

[9] Dobrowolski T., Kink Motion in a Curved Josephson Junction, Phys. Rev. E 79 (2009) 046601 [7 pp].

[10] Dobrowolski T., The Studies on the Motion of the Sine-Gordon Kink on a Curved Surface, Ann. Phys. (Berlin) 522 (2010) 574-583.

[11] Ekert A. and Jozsa R., Quantum Computation and Shor's Factoring Algorithm, Rev. Mod. Phys. 68 (1996) 733-753.

[12] Ferreira L., Piette B. and Zakrzewski W., Wobbles and Other Kink-Breather Solutions of the Sine-Gordon Model, Phys. Rev. E 77 (2008) 036613 [9 pp].

[13] Friedman J., Patel V., Chen W., Tolpygo S. and Lukens J., Quantum Superposition of Distinct Macroscopic States, Nature 406 (2000) 43-46.

[14] Gorria C., Gaididei Yu., Soerensen M., Christiansen P. and Caputo J., Kink Propagation and Trapping in a Two-Dimensional Curved Josephson Junction, Phys. Rev. B 69 (2004) 134506 [10 pp].

[15] Grønbech-Jensen N., Lomdahl P., and Samuelsen M., Phase-Locking of Long Annular Josephson Junctions Coupled to an External RF Magnetic Field, Phys. Lett. A 154 (1991) 14-18. 
[16] Josephson B., Possible New Effects in Superconductive Tunnelling, Phys. Lett. 1 (1962) 251-253.

[17] Josephson B., Supercurrents Through Barriers, Adv. Phys. 14 (1965) 419451.

[18] Kemp A., Wallraff A. and Ustinov A., Josephson Vortex Qubit: Design, Preparation and Read-Out, Phys. Stat. Sol. B 233 (2002) 472-481.

[19] Nakamura Y., Pashkin Yu. and Tsai J., Coherent Control of Macroscopic Quantum States in a Single-Cooperpair Box, Nature 398 (1999) 786-788.

[20] Ogawa N., Curvature-Dependent Diffusion Flow on a Surface with Thickness, Phys. Rev. E 81 (2010) 061113 [8 pp].

[21] Rice M., Physical Dynamics of Solitons, Phys. Rev. B 28 (1983) 3587-3589.

[22] Swihart J., Field Solution for a Thin-Film Superconducting Strip Transmission Line, J. Appl. Phys. 32 (1961) 461-469.

[23] Ustinov A., Malomed B., and N. Thyssen N., Soliton Trapping in a Periodic Potential: Experiment, Phys. Lett. A 233 (1997) 239-244.

[24] van der Wal C., ter Haar A., Wilhelm F., Schouten R., Harmans C., Orlando T., Lloyd S. and Mooij J., Quantum Superposition of Macroscopic PersistentCurrent States, Science 290 (2000) 773-777.

Tomasz Dobrowolski

Institute of Physics UP

Podchorążych 2

30-084 Kraków, POLAND

E-mail address: sfdobrow@cyf-kr.edu.pl 